\title{
A Simplified Analysis on a Pulse Detonation Engine Model
}

\author{
By Takuma ENDO ${ }^{1)}$ and Toshi FUJIWARA ${ }^{2)}$ \\ 1) Center for Integrated Research in Science and Engineering, Nagoya University, Nagoya, Japan \\ ${ }^{2)}$ Department of Aerospace Engineering, Nagoya University, Nagoya, Japan
}

(Received April 19th, 2001)

\begin{abstract}
The performance of pulse detonation engines was analytically estimated by using a simple model. A pulse detonation engine was modeled as a straight tube. One end of the tube was closed and the other was open, and a detonation wave was ignited at the closed end. One cycle of the pulse-detonation-engine operation was divided into three phases: combustion, exhaust, and filling phases. The combustion and exhaust phases were theoretically analyzed with some simplifications, using the Hugoniot relation for the Chapman-Jouguet detonation wave and flow relations for self-similar rarefaction waves. Based on the simplified theoretical analysis, useful formulas for impulse density per one-cycle operation and time-averaged thrust density were derived.
\end{abstract}

Key Words: Pulse Detonation Engine, Theoretical Analysis, Gas Dynamics

\section{Nomenclature}

$a: \quad$ sonic speed of gas

$c_{v}: \quad$ specific heat at constant volume per unit mass

$D$ : propagation velocity of specified surface in $x$ coordinate

$D_{\mathrm{CJ}}$ : Chapman-Jouguet detonation speed of detonable mixture

$f_{\text {cyc }}$ : frequency of cyclic pulse-detonation-engine operation

$I_{\text {cyc }}$ impulse density acting on thrust wall per one-cycle operation

$L: \quad$ length of pulse detonation engine

$\ell_{\mathrm{f}}$ : characteristic length of detonable-mixture injection

$M_{\mathrm{CJ}}$ : Mach number of Chapman-Jouguet detonation wave $\left(=D_{\mathrm{CJ}} / a_{1}\right)$

$p:$ thermodynamic pressure of gas

$p_{\text {av }}:$ time-averaged thrust density

$p_{\mathrm{w}}(t)$ : pressure acting on thrust wall as a function of time

$q:$ heat released in chemical reaction per unit mass

$R_{\mathrm{u}}$ : universal gas constant

$T:$ thermodynamic temperature of gas

$t$ : time relative to instant of ignition

$T_{\text {cyc }}$ : period of cyclic pulse-detonation-engine operation

$t_{\mathrm{I}}:$ time at which $x_{3}$ becomes $L$

$t_{\mathrm{II}}$ : time at which front boundary of rarefaction wave originating from open end reaches closed end

$t_{\mathrm{III}}$ : time at which exhaust of burned gas is completed

$u$ : flow velocity of gas in $x$ coordinate

$V_{\mathrm{f}}$ : characteristic speed of detonable-mixture injection

$x$ : coordinate along axis of pulse detonation engine, where $x=0$ and $x=L$ correspond to closed and open ends, respectively $\gamma:$ specific-heat ratio of gas

$\mu: \quad$ average molecular weight of gas

$\rho:$ mass density of gas

$\tau_{\mathrm{f}}:$ duration of filling phase

Subscripts

1: undisturbed detonable mixture

2: Chapman-Jouguet surface of detonation wave

3 : rear boundary of rarefaction wave following detonation wave

ex : open-end boundary of rarefaction wave originating from open end

$\mathrm{N}$ : von Neumann spike of detonation wave

\section{Introduction}

A pulse detonation engine (PDE) is expected to be a highperformance next-generation aerospace propulsion engine. In PDEs, fuel is burned as a self-sustaining detonation wave propagating through a tube. The combustion as a detonation wave is close to an isochoric process rather than an isobaric one. Therefore higher heat-cycle efficiency is expected for PDEs than for conventional aerospace propulsion engines, in which combustion is close to an isobaric process. ${ }^{1)}$

Although research on PDEs has more than a half-century of history, interests in them have been renewed in the past several years. Among the worldwide evolution of PDE researches, a comprehensive review on propulsion applications of detonation waves was recently published. ${ }^{2)}$ A great part of current PDE research seems to be more practical than fundamental, seriously aiming at the commercial use of PDEs.

Because PDEs are of simple structures, performance estimations on them are carried out mostly by methods of computational fluid dynamics (CFD). It is true that performance estimations by CFD methods are more accurate than those by simplified analytical methods, analytical methods 
are often more suitable for understanding the performance of PDEs in perspective. Although Bussing and Pappas ${ }^{1)}$ already showed an analytical treatment for performance estimations on PDEs, their treatment was not adequate enough to elucidate the dependence of PDE performance on governing parameters. In this paper, a simple performance analysis on PDEs is presented. Using the Hugoniot relation for the Chapman-Jouguet (CJ) detonation wave and flow relations for self-similar rarefaction waves, pressure on the thrust wall is estimated as a function of time. Based on the analysis, some useful formulas are derived.

\section{Model}

A PDE is modeled as a straight tube with a constant cross section. One end of the tube is closed and the other is open. For simplicity, gases are treated as polytropic gases. ${ }^{3)}$ And it is also assumed that the viscous effects and thermal conduction are negligible and that flow is one-dimensional. In the analysis below, one cycle of PDE operation is examined.

Initially, the tube is filled with a uniform detonable mixture at rest, as shown in Fig. 1(a). The initial undisturbed mixture is characterized by $\rho_{1}, p_{1}, \gamma_{1}, u_{1}(=0)$. At time $t=0$, it is ignited at $x=0$, and the CJ detonation wave starts to propagate from the closed end to the open one. The initiation process of the CJ detonation wave is among the most important issues for PDEs. ${ }^{4)}$ But in this model, this issue is not treated, and it is assumed that the CJ detonation wave starts to propagate from the closed end, namely $x=0$, at $t=0$.

By the passing of the $\mathrm{CJ}$ detonation wave through the gas, the gas is accelerated in the same direction as the wave propagation because the $\mathrm{CJ}$ detonation wave is a compression wave. Since the gas attached to the closed end should be at rest, the gas between the $\mathrm{CJ}$ detonation wave and the closed end is decelerated. This deceleration is realized in a selfsimilar rarefaction wave following the $\mathrm{CJ}$ detonation wave. This situation is shown in Fig. 1(b). The front boundary of the rarefaction wave coincides with the CJ surface of the detonation wave. The gas at the CJ surface of the detonation wave is characterized by $\rho_{2}, p_{2}, \gamma_{2}, u_{2}$. The rear boundary of the rarefaction wave also propagates toward the open end. The gas at the rear boundary of the rarefaction wave is characterized by $\rho_{3}, p_{3}, \gamma_{3}\left(=\gamma_{2}\right), u_{3}(=0)$, which also characterize the whole gas in region $0 \leq x \leq x_{3}$. In this model, $x_{3}$ becomes $L$ at time $t=t_{\mathrm{I}}$, and at this instant, all gas in the tube is characterized by $\rho_{3}, p_{3}, \gamma_{3}\left(=\gamma_{2}\right), u_{3}(=0)$, as shown in Fig. 1(c). The period $0 \leq t \leq t_{\mathrm{I}}$ is named the combustion phase.

At time $t=t_{\mathrm{I}}$, another rarefaction wave is created and starts to propagate from the open end to the closed one. The front boundary of this rarefaction wave propagates at sonic speed $a_{3}$, and reaches the closed end at $t=t_{\mathrm{II}}$. Figures $1(\mathrm{~d})$ and 1(e) show this situation. Through this rarefaction wave, the burned gas is exhausted from the open end of the tube. The exhaust of the burned gas lasts until time $t=t_{\mathrm{III}}$, as

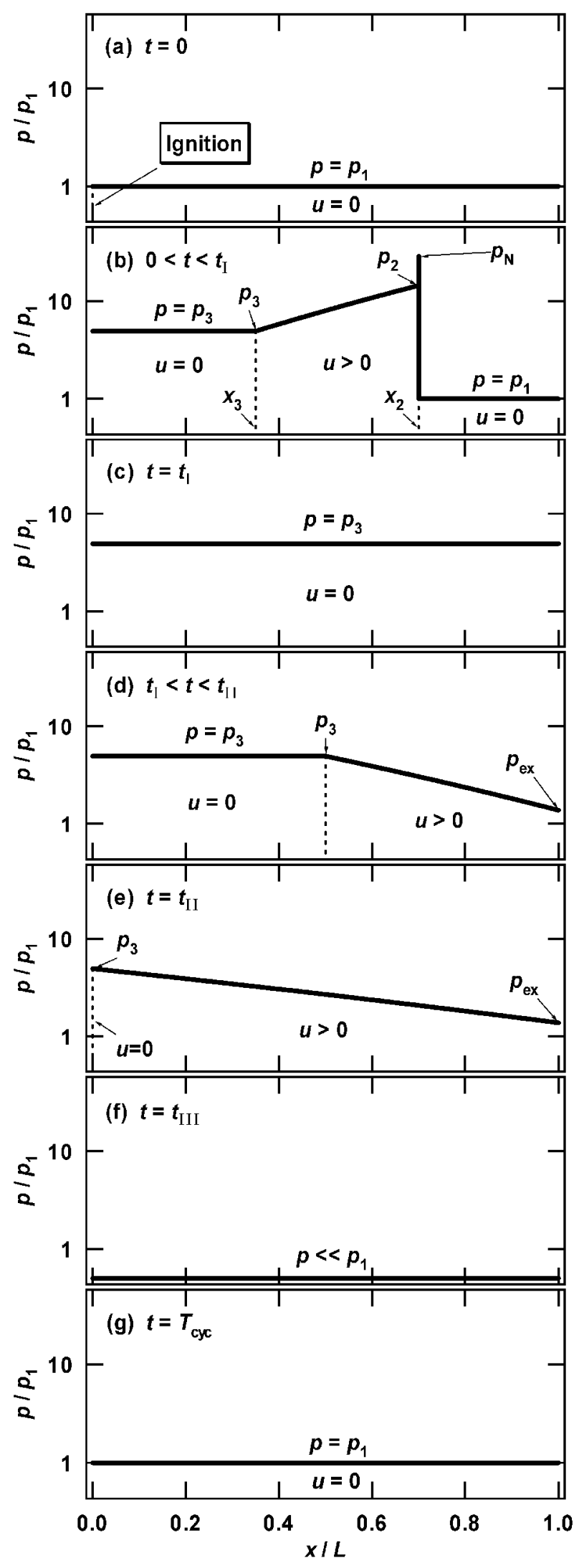

Fig. 1. Pressure distributions at some instants: (a) $t=0$, (b) $0<t<t_{\mathrm{I}}$, (c) $t=t_{\mathrm{I}}$, (d) $t_{\mathrm{I}}<t<t_{\mathrm{II}}$, (e) $t=t_{\mathrm{II}}$, (f) $t=t_{\mathrm{III}}$, (g) $t=T_{\text {cyc }}$. Calculation was carried out by using a typical parameter set the same as that used in "numerical example" of the text. 
shown in Fig. 1(f). The period $t_{\mathrm{I}} \leq t \leq t_{\mathrm{III}}$ is named the exhaust phase. After time $t=t_{\mathrm{III}}$, the tube is recharged with a fresh detonable mixture, and the recharging of the tube is completed at time $t=T_{\text {cyc }}$, shown in Fig. 1(g). Namely, $T_{\text {cyc }}$ is the period of the cyclic PDE operation. The period $t_{\mathrm{III}} \leq t \leq T_{\text {cyc }}$ is named the filling phase.

\section{Analysis}

First, the quantities on the CJ surface $\rho_{2}, p_{2}, u_{2}, D_{2}$ are examined. When the heat released in the chemical reaction per unit mass $q$ is much larger than the internal thermal energy per unit mass of the unburned gas $c_{v 1} T_{1}$, they are written by the Hugoniot relation as follows, ${ }^{5}$ )

$$
\begin{aligned}
\rho_{2} & =\frac{\gamma_{2}+1}{\gamma_{2}} \rho_{1}, \\
p_{2} & =\frac{\gamma_{1}}{\gamma_{2}+1} M_{\mathrm{CJ}}^{2} p_{1}, \\
u_{2} & =\frac{1}{\gamma_{2}} a_{2}=\frac{1}{\gamma_{2}+1} D_{\mathrm{CJ}}, \\
D_{2} & =D_{\mathrm{CJ}}=\sqrt{2\left(\gamma_{2}^{2}-1\right) q} .
\end{aligned}
$$

Since thickness of the CJ detonation wave is generally negligible compared with tube length, the position of the CJ surface is written by

$$
x_{2}=D_{2} t=D_{\mathrm{CJ}} t .
$$

On the von Neumann spike, $\rho_{\mathrm{N}}$ and $p_{\mathrm{N}}$ are written as follows, ${ }^{3)}$

$$
\begin{aligned}
& \rho_{\mathrm{N}}=\frac{\gamma_{1}+1}{\gamma_{1}-1} \rho_{1}, \\
& p_{\mathrm{N}}=\frac{2 \gamma_{1}}{\gamma_{1}+1} M_{\mathrm{CJ}}^{2} p_{1} .
\end{aligned}
$$

Second, the rarefaction wave following the CJ detonation wave is examined. This wave is a self-similar rarefaction wave whose front boundary coincides with the CJ surface. Therefore the state of the gas at the front boundary is given by $\rho_{2}, p_{2}, \gamma_{2}, u_{2}, D_{2}$. The state of the flow inside the rarefaction wave is written by the relation for the self-similar rarefaction wave as follows, ${ }^{6}$ )

$$
\begin{aligned}
& \rho=\left(\frac{1}{\gamma_{2}}+\frac{\gamma_{2}-1}{\gamma_{2}} \frac{x}{x_{2}}\right)^{\frac{2}{\gamma_{2}-1}} \rho_{2}, \\
& p=\left(\frac{1}{\gamma_{2}}+\frac{\gamma_{2}-1}{\gamma_{2}} \frac{x}{x_{2}}\right)^{\frac{2 \gamma_{2}}{\gamma_{2}-1}} p_{2}, \\
& u=u_{2}-\frac{2}{\gamma_{2}+1} \frac{x_{2}-x}{t}=-a+\frac{x}{t}\left(\leq \frac{1}{\gamma_{2}} a\right), \\
& a=a_{2}-\frac{\gamma_{2}-1}{\gamma_{2}+1} \frac{x_{2}-x}{t} .
\end{aligned}
$$

At the rear boundary of the rarefaction wave, the flow velocity is zero:

$$
u_{3}=0 \text {. }
$$

Therefore the state of the gas at the rear boundary of the rarefaction wave is given as follows,

$$
\begin{aligned}
& x_{3}=\frac{1}{2} x_{2}, \\
& D_{3}=a_{3}=\frac{1}{2} D_{\mathrm{CJ}}, \\
& \rho_{3}=2\left(\frac{\gamma_{2}+1}{2 \gamma_{2}}\right)^{\frac{\gamma_{2}+1}{\gamma_{2}-1}} \rho_{1}, \\
& p_{3}=\frac{\gamma_{1}}{2 \gamma_{2}}\left(\frac{\gamma_{2}+1}{2 \gamma_{2}}\right)^{\frac{\gamma_{2}+1}{\gamma_{2}-1}} M_{\mathrm{CJ}}^{2} p_{1} .
\end{aligned}
$$

Although these solutions are not exactly valid after the time when the detonation wave exits the open end of the tube, an estimation

$$
t_{\mathrm{I}}=\frac{L}{D_{3}}=\frac{2 L}{D_{\mathrm{CJ}}}
$$

is adopted for simplicity. And at time $t=t_{\mathrm{I}}$, all gas in the tube is considered to be characterized by $\rho_{3}, p_{3}, \gamma_{3}\left(=\gamma_{2}\right)$, $u_{3}(=0)$. In the combustion phase $0 \leq t \leq t_{\mathrm{I}}$, the pressure acting on the thrust wall $p_{\mathrm{w}}(t)$ is given by $p_{3}$.

Third, the exhaust phase is examined. At time $t=t_{\mathrm{I}}$, another rarefaction wave is created and starts to propagate through the tube from the open end to the closed one. The front boundary of the rarefaction wave propagates with sonic speed $a_{3}$ in the $-x$ direction. Therefore the time required for the propagation of the rarefaction wave from the open end to the closed one is estimated by

$$
t_{\mathrm{II}}-t_{\mathrm{I}}=\frac{L}{a_{3}}=\frac{2 L}{D_{\mathrm{CJ}}} .
$$

Therefore, $t_{\mathrm{II}}$ is given by

$$
t_{\mathrm{II}}=\frac{2 L}{D_{\mathrm{CJ}}}+t_{\mathrm{I}}=\frac{4 L}{D_{\mathrm{CJ}}} .
$$

The state of the gas inside the rarefaction wave is written by the relation for the self-similar rarefaction wave as follows, ${ }^{6}$ )

$$
\begin{aligned}
& \rho=\left(1+\frac{\gamma_{2}-1}{D_{\mathrm{CJ}}} \frac{L-x}{t-t_{\mathrm{I}}}\right)^{\frac{2}{\gamma_{2}-1}} \rho_{\mathrm{ex}}, \\
& p=\left(1+\frac{\gamma_{2}-1}{D_{\mathrm{CJ}}} \frac{L-x}{t-t_{\mathrm{I}}}\right)^{\frac{2 \gamma_{2}}{\gamma_{2}-1}} p_{\mathrm{ex}}, \\
& u=u_{\mathrm{ex}}-\frac{2}{\gamma_{2}+1} \frac{L-x}{t-t_{\mathrm{I}}}=a-\frac{L-x}{t-t_{\mathrm{I}}}(\leq a), \\
& a=a_{\mathrm{ex}}+\frac{\gamma_{2}-1}{\gamma_{2}+1} \frac{L-x}{t-t_{\mathrm{I}}},
\end{aligned}
$$

where

$$
\begin{aligned}
& \rho_{\mathrm{ex}}=\frac{\gamma_{2}+1}{\gamma_{2}^{\frac{\gamma_{2}+1}{\gamma_{2}-1}}} \rho_{1}, \\
& p_{\mathrm{ex}}=\frac{\gamma_{1}}{\gamma_{2}{ }^{\frac{2 \gamma_{2}}{\gamma_{2}-1}}\left(\gamma_{2}+1\right)} M_{\mathrm{CJ}}^{2} p_{1}, \\
& u_{\mathrm{ex}}=a_{\mathrm{ex}}=\frac{1}{\gamma_{2}+1} D_{\mathrm{CJ}}
\end{aligned}
$$


stand for the state of the gas at the open end during $t_{\mathrm{I}} \leq t \leq t_{\mathrm{II}}$. Using these solutions, the mass exhaust rate per unit area is given by

$$
\rho_{\mathrm{ex}} u_{\mathrm{ex}}=\frac{2^{\frac{2}{\gamma_{2}-1}}}{\left(\gamma_{2}+1\right)^{\frac{\gamma_{2}+1}{\gamma_{2}-1}}} \rho_{3} D_{\mathrm{CJ}}=\left(\frac{2}{\gamma_{2}+1}\right)^{\frac{\gamma_{2}+1}{\gamma_{2}-1}} \rho_{3} a_{3} .
$$

At time $t=t_{\mathrm{I}}$ when the exhausting rarefaction wave starts to propagate, the total mass of the gas per unit area in the tube is $\rho_{3} L$. When $\rho_{\mathrm{ex}} u_{\mathrm{ex}}$ is considered to be the characteristic mass exhaust rate per unit area, the period of the exhaust phase is estimated as

$t_{\mathrm{III}}-t_{\mathrm{I}}=\frac{\rho_{3} L}{\rho_{\mathrm{ex}} u_{\mathrm{ex}}}=\frac{\left(\gamma_{2}+1\right)^{\frac{\gamma_{2}+1}{\gamma_{2}-1}}}{2^{\frac{2}{\gamma_{2}-1}}} \frac{L}{D_{\mathrm{CJ}}}=\left(\frac{\gamma_{2}+1}{2}\right)^{\frac{\gamma_{2}+1}{\gamma_{2}-1}} \frac{L}{a_{3}}$.

During $t_{\mathrm{I}} \leq t \leq t_{\mathrm{II}}$ in this phase, the front boundary of the exhausting rarefaction wave does not reach the closed end; therefore the pressure acting on the thrust wall $p_{\mathrm{w}}(t)$ remains $p_{3}$. On the assumption of temporally linear decay of the thrust during $t_{\mathrm{II}} \leq t \leq t_{\mathrm{III}}, p_{\mathrm{W}}(t)$ is written as

$$
p_{\mathrm{w}}\left(t ; t_{\mathrm{II}} \leq t \leq t_{\mathrm{III}}\right)=p_{3}\left(1-\frac{t-t_{\mathrm{II}}}{t_{\mathrm{III}}-t_{\mathrm{II}}}\right),
$$

where

$$
t_{\mathrm{III}}=\left[1+\left(\frac{\gamma_{2}+1}{2}\right)^{\frac{\gamma_{2}+1}{\gamma_{2}-1}}\right] \frac{2 L}{D_{\mathrm{CJ}}} .
$$

Here the filling phase and some important quantities are discussed. The period of the cyclic PDE operation $T_{\text {cyc }}$ is written as

$$
\begin{aligned}
T_{\mathrm{cyc}}=t_{\mathrm{III}}+\tau_{\mathrm{f}} & =\left[1+\left(\frac{\gamma_{2}+1}{2}\right)^{\frac{\gamma_{2}+1}{\gamma_{2}-1}}\right] \frac{2 L}{D_{\mathrm{CJ}}}+\tau_{\mathrm{f}} \\
& >\left[1+\left(\frac{\gamma_{2}+1}{2}\right)^{\frac{\gamma_{2}+1}{\gamma_{2}-1}}\right] \frac{2 L}{D_{\mathrm{CJ}}},
\end{aligned}
$$

where $\tau_{\mathrm{f}}>0$ was used. And thus the frequency of the cyclic PDE operation $f_{\text {cyc }}$ is written as

$$
\begin{aligned}
f_{\text {cyc }}=\frac{1}{T_{\text {cyc }}} & =\frac{1}{\left[1+\left(\frac{\gamma_{2}+1}{2}\right)^{\frac{\gamma_{2}+1}{\gamma_{2}-1}}\right] \frac{2 L}{D_{\mathrm{CJ}}}+\tau_{\mathrm{f}}} \\
& <\frac{1}{\left[1+\left(\frac{\gamma_{2}+1}{2}\right)^{\frac{\gamma_{2}+1}{\gamma_{2}-1}}\right] \frac{2 L}{D_{\mathrm{CJ}}}} .
\end{aligned}
$$

Since the thrust density as a function of time in this simplified model is summarized as

$$
p_{\mathrm{w}}(t)=\left\{\begin{array}{ll}
p_{3} & \left(0 \leq t \leq t_{\mathrm{II}}\right) \\
p_{3}\left(1-\frac{t-t_{\mathrm{II}}}{t_{\mathrm{III}}-t_{\mathrm{II}}}\right) & \left(t_{\mathrm{II}} \leq t \leq t_{\mathrm{III}}\right) \\
0 & \left(t_{\mathrm{III}} \leq t \leq T_{\mathrm{cyc}}\right)
\end{array},\right.
$$

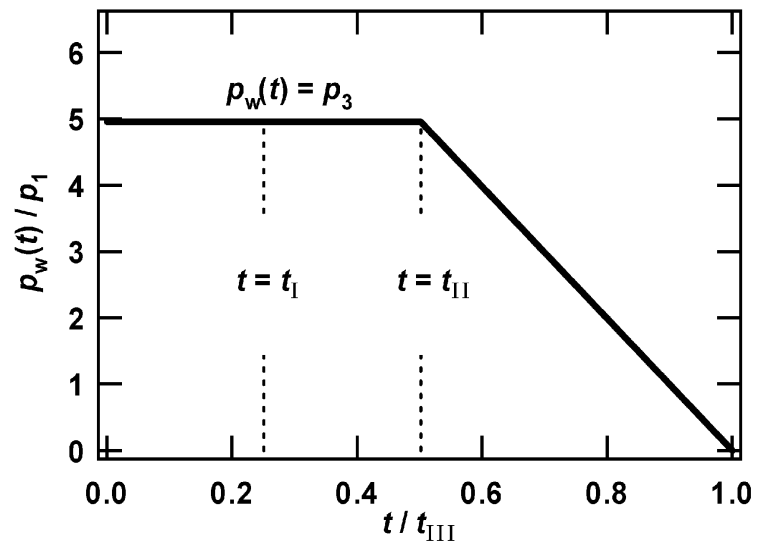

Fig. 2. Pressure acting on the thrust wall as a function of time. Calculation was carried out by using a typical parameter set the same as that used in "numerical example" of the text.

which is shown in Fig. 2, the impulse density acting on the thrust wall per one-cycle operation $I_{\text {cyc }}$ is written as follows,

$$
\begin{aligned}
I_{\mathrm{cyc}} & =\int_{0}^{T_{\mathrm{cyc}}} p_{\mathrm{w}}(t) d t \\
& =\int_{0}^{t_{\mathrm{II}}} p_{3} d t+\int_{t_{\mathrm{II}}}^{t_{\mathrm{III}}} p_{3}\left(1-\frac{t-t_{\mathrm{II}}}{t_{\mathrm{III}}-t_{\mathrm{II}}}\right) d t \\
& =\frac{1}{2 \gamma_{2}}\left(\frac{\gamma_{2}+1}{2 \gamma_{2}}\right)^{\frac{\gamma_{2}+1}{\gamma_{2}-1}}\left[3+\left(\frac{\gamma_{2}+1}{2}\right)^{\frac{\gamma_{2}+1}{\gamma_{2}-1}}\right] \rho_{1} D_{\mathrm{CJ}} L .
\end{aligned}
$$

In this solution, $a_{1}^{2}=\gamma_{1} p_{1} / \rho_{1}$ was used. Qualitatively, the pressure acting on the thrust wall is proportional to $\left(D_{\mathrm{CJ}} / a_{1}\right)^{2} p_{1}$, and the duration for which this wall is pushed by the thermodynamic gas pressure is proportional to $L / D_{\mathrm{CJ}}$; therefore dependency of the impulse is

$$
I_{\text {cyc }} \propto\left(\frac{D_{\mathrm{CJ}}}{a_{1}}\right)^{2} p_{1} \frac{L}{D_{\mathrm{CJ}}} \propto \frac{D_{\mathrm{CJ}}^{2}}{\frac{p_{1}}{\rho_{1}}} p_{1} \frac{L}{D_{\mathrm{CJ}}} \propto \rho_{1} D_{\mathrm{CJ}} L .
$$

When the equation of state for an ideal gas,

$$
p_{1}=\rho_{2} \frac{R_{\mathrm{u}}}{\mu_{1}} T_{1},
$$

is used, $I_{\text {cyc }}$ is written as follows,

$I_{\mathrm{cyc}}=\frac{1}{2 \gamma_{2}}\left(\frac{\gamma_{2}+1}{2 \gamma_{2}}\right)^{\frac{\gamma_{2}+1}{\gamma_{2}-1}}\left[3+\left(\frac{\gamma_{2}+1}{2}\right)^{\frac{\gamma_{2}+1}{\gamma_{2}-1}}\right] \frac{p_{1} \mu_{1}}{R_{\mathrm{u}} T_{1}} D_{\mathrm{CJ}} L$.

Furthermore, the time-averaged thrust density $p_{\text {av }}$ is written as follows, 


$$
\begin{aligned}
p_{\mathrm{av}} & =\frac{I_{\mathrm{cyc}}}{T_{\mathrm{cyc}}} \\
& =\frac{\frac{1}{2 \gamma_{2}}\left(\frac{\gamma_{2}+1}{2 \gamma_{2}}\right)^{\frac{\gamma_{2}+1}{\gamma_{2}-1}}\left[3+\left(\frac{\gamma_{2}+1}{2}\right)^{\frac{\gamma_{2}+1}{\gamma_{2}-1}}\right] \rho_{1} D_{\mathrm{CJ}} L}{\left[1+\left(\frac{\gamma_{2}+1}{2}\right)^{\frac{\gamma_{2}+1}{\gamma_{2}-1}}\right] \frac{2 L}{D_{\mathrm{CJ}}}+\tau_{\mathrm{f}}} \\
& <\frac{1}{4 \gamma_{2}}\left(\frac{\gamma_{2}+1}{2 \gamma_{2}}\right)^{\frac{\gamma_{2}+1}{\gamma_{2}-1}} \frac{3+\left(\frac{\gamma_{2}+1}{2}\right)^{\frac{\gamma_{2}+1}{\gamma_{2}-1}}}{1+\left(\frac{\gamma_{2}+1}{2}\right)^{\frac{\gamma_{2}+1}{\gamma_{2}-1}} \rho_{1} D_{\mathrm{CJ}}{ }^{2} .}
\end{aligned}
$$

When the equation of state for an ideal gas is used, it is written as follows,

$$
\begin{gathered}
p_{\mathrm{av}}=\frac{\frac{1}{2 \gamma_{2}}\left(\frac{\gamma_{2}+1}{2 \gamma_{2}}\right)^{\frac{\gamma_{2}+1}{\gamma_{2}-1}}\left[3+\left(\frac{\gamma_{2}+1}{2}\right)^{\frac{\gamma_{2}+1}{\gamma_{2}-1}}\right] \frac{p_{1} \mu_{1}}{R_{\mathrm{u}} T_{1}} D_{\mathrm{CJ}} L}{\left[1+\left(\frac{\gamma_{2}+1}{2}\right)^{\frac{\gamma_{2}+1}{\gamma_{2}-1}}\right] \frac{2 L}{D_{\mathrm{CJ}}}+\tau_{\mathrm{f}}} \\
<\frac{1}{4 \gamma_{2}}\left(\frac{\gamma_{2}+1}{2 \gamma_{2}}\right)^{\frac{\gamma_{2}+1}{\gamma_{2}-1}} \frac{3+\left(\frac{\gamma_{2}+1}{2}\right)^{\frac{\gamma_{2}+1}{\gamma_{2}-1}}}{1+\left(\frac{\gamma_{2}+1}{2}\right)^{\frac{\gamma_{2}+1}{\gamma_{2}-1}} \frac{p_{1} \mu_{1}}{R_{\mathrm{u}} T_{1}} D_{\mathrm{CJ}}{ }^{2} .}
\end{gathered}
$$

The shorter the duration of filling phase $\tau_{\mathrm{f}}$, the larger the time-averaged thrust density $p_{\text {av }}$. This is to be expected because the filling phase is a dead phase of a PDE operation cycle. The duration of filling phase $\tau_{\mathrm{f}}$ can be estimated by $\ell_{\mathrm{f}} / V_{\mathrm{f}}$. Generally in PDEs, tube diameter is much shorter than tube length. Therefore the detonable-mixture injection in the radial direction rather than the axial direction may be effective in shortening the duration of the filling phase.

\section{Numerical Example}

We show a numerical example by using a typical parameter set. First we use $\gamma_{1}=\gamma_{2}=7 / 5$. In this case, following formulas are obtained.

$$
\begin{aligned}
& \rho_{2}=\frac{12}{7} \rho_{1} \\
& p_{2} \approx\left(\frac{M_{\mathrm{CJ}}}{1.3}\right)^{2} p_{1} \\
& u_{2}=\frac{5}{7} a_{2}=\frac{5}{12} D_{\mathrm{CJ}} \\
& \rho_{\mathrm{N}}=6 \rho_{1}
\end{aligned}
$$

$$
\begin{aligned}
& p_{\mathrm{N}} \approx\left(\frac{M_{\mathrm{CJ}}}{0.93}\right)^{2} p_{1} \\
& \rho_{3} \approx 0.79 \rho_{1} \\
& p_{3} \approx\left(\frac{M_{\mathrm{CJ}}}{2.2}\right)^{2} p_{1} \\
& u_{3}=0 \\
& a_{3}=D_{3}=\frac{1}{2} D_{\mathrm{CJ}} \\
& \rho_{\mathrm{ex}} \approx 0.32 \rho_{1} \\
& p_{\mathrm{ex}} \approx\left(\frac{M_{\mathrm{CJ}}}{4.3}\right)^{2} p_{1} \\
& u_{\mathrm{ex}}=a_{\mathrm{ex}}=\frac{5}{12} D_{\mathrm{CJ}} \\
& \rho_{\mathrm{ex}} u_{\mathrm{ex}} \approx 0.17 \rho_{3} D_{\mathrm{CJ}} \approx 0.33 \rho_{3} a_{3} \\
& t_{\mathrm{I}}=2 \frac{L}{D_{\mathrm{CJ}}} \\
& t_{\mathrm{cyc}} \approx 8.0 \frac{L}{D_{\mathrm{CJ}}}+t_{\mathrm{I}} \approx 6.0 \frac{L}{D_{\mathrm{CJ}}} \\
& t_{\mathrm{II}}=4 \frac{L}{D_{\mathrm{CJ}}} \\
& t_{\mathrm{III}} \approx 8.0 \frac{L}{D_{\mathrm{CJ}}}+\tau_{\mathrm{CJ}} \\
& t_{\mathrm{fJ}}
\end{aligned}
$$

Furthermore, we use $D_{\mathrm{CJ}}=2000 \mathrm{~m} / \mathrm{s}, a_{1}=400 \mathrm{~m} / \mathrm{s}$, $p_{1}=10^{5} \mathrm{~Pa}$, and $L=2 \mathrm{~m}$ as a typical parameter set, which corresponds to $\rho_{1}=\gamma_{1} p_{1} / a_{1}{ }^{2}=0.875 \mathrm{~kg} / \mathrm{m}^{3}$. In this case, the following values are obtained.

$$
\begin{aligned}
& \rho_{2}=1.5 \mathrm{~kg} / \mathrm{m}^{3} \\
& p_{2} \approx 15 p_{1} \approx 15 \times 10^{5} \mathrm{~Pa} \\
& u_{2} \approx 830 \mathrm{~m} / \mathrm{s} \\
& a_{2} \approx 1200 \mathrm{~m} / \mathrm{s} \\
& \rho_{\mathrm{N}}=5.25 \mathrm{~kg} / \mathrm{m}^{3} \\
& p_{\mathrm{N}} \approx 29 p_{1} \approx 29 \times 10^{5} \mathrm{~Pa} \\
& \rho_{3} \approx 0.69 \mathrm{~kg} / \mathrm{m}^{3} \\
& p_{3} \approx 5.0 p_{1} \approx 5.0 \times 10^{5} \mathrm{~Pa}
\end{aligned}
$$




$$
\begin{aligned}
& u_{3}=0 \\
& a_{3}=D_{3}=1000 \mathrm{~m} / \mathrm{s} \\
& \rho_{\mathrm{ex}} \approx 0.28 \mathrm{~kg} / \mathrm{m}^{3} \\
& p_{\mathrm{ex}} \approx 1.4 p_{1} \approx 1.4 \times 10^{5} \mathrm{~Pa} \\
& u_{\mathrm{ex}}=a_{\mathrm{ex}} \approx 830 \mathrm{~m} / \mathrm{s} \\
& \rho_{\mathrm{ex}} u_{\mathrm{ex}} \approx 230 \mathrm{~kg} /\left(\mathrm{m}^{2} \cdot \mathrm{s}\right) \\
& t_{\mathrm{I}}=2.0 \mathrm{~ms} \\
& t_{\mathrm{II}}=4.0 \mathrm{~ms} \\
& t_{\mathrm{III}} \approx 8.0 \mathrm{~ms} \\
& T_{\mathrm{cyc}} \approx 8.0[\mathrm{~ms}]+\tau_{\mathrm{f}}>8.0 \mathrm{~ms} \\
& f_{\mathrm{cyc}} \approx \frac{1}{8.0[\mathrm{~ms}]+\tau_{\mathrm{f}}}<130 \mathrm{~Hz} \\
& I_{\mathrm{cyc}} \approx 3000(\mathrm{~N} \cdot \mathrm{s}) / \mathrm{m}^{2} \\
& p_{\mathrm{av}} \approx \frac{30}{8.0+\tau_{\mathrm{f}}[\mathrm{ms}]} \times 10^{5} \mathrm{~Pa}<3.7 \times 10^{5} \mathrm{~Pa} .
\end{aligned}
$$

If $p_{\mathrm{ex}}$ is less than pressure outside the tube, the treatment of the exhaust phase in this paper is invalid. Initial pressure $p_{1}$ is generally not less than pressure outside the tube, and $p_{\mathrm{ex}}$ was estimated to be $p_{\text {ex }} \approx 1.4 p_{1}$ in this example; therefore the above estimation of the exhaust phase is valid.

\section{Conclusions}

We analytically obtained simple formulas for the performance of pulse detonation engines (PDEs). A PDE was modeled as a straight tube. One end was closed and the other was open; a detonation wave was ignited at the closed end. We calculated pressure acting on the closed end, with some simplifications, using the Hugoniot relation for the Chapman-Jouguet (CJ) detonation wave and flow relations for self-similar rarefaction waves. The most important formula obtained in this paper is $p_{\mathrm{av}} \approx\left(0.85 \rho_{1} D_{\mathrm{CJ}} L\right) /\left[\left(8.0 L / D_{\mathrm{CJ}}\right)+\tau_{\mathrm{f}}\right]<0.11 \rho_{1} D_{\mathrm{CJ}}{ }^{2}$ for the specific-heat ratio of $7 / 5$, where $p_{\mathrm{av}}$ is the time-averaged thrust density; $\rho_{1}$ and $D_{\mathrm{CJ}}$ are, respectively, the mass density and $\mathrm{CJ}$ detonation speed of the detonable mixture; $L$ is the length of the tube; and $\tau_{\mathrm{f}}$ is the time required to fill the tube with a fresh detonable mixture.

\section{Acknowledgment}

The authors sincerely acknowledge valuable discussions with Dr. T. Miyasaka and Mr. K. Murakami.

\section{References}

1) Bussing, T. and Pappas, G.: An Introduction to Pulse Detonation Engines, AIAA Paper 94-0263, 1994.

2) Kailasanath, K.: Review of Propulsion Applications of Detonation Waves, AIAA J., 38 (2000), pp. 1698-1708.

3) Landau, L. D. and Lifshitz, E. M.: Fluid Mechanics, 2nd ed., Butterworth-Heinemann, Oxford, 1987, Ch. 9.

4) Lee, J. H. S. and Moen, I. O.: The Mechanism of Transition from Deflagration to Detonation in Vapor Cloud Explosions, Prog. Energy Combust. Sci., 6 (1980), pp. 359-389.

5) Landau, L. D. and Lifshitz, E. M.: Fluid Mechanics, 2nd ed., Butterworth-Heinemann, Oxford, 1987, Ch. 14.

6) Landau, L. D. and Lifshitz, E. M.: Fluid Mechanics, 2nd ed., Butterworth-Heinemann, Oxford, 1987, Ch. 10. 